\title{
Electroweak penguin decays
}

\author{
Jacco Andreas de $\operatorname{Vries}^{1 a, *}$ \\ ${ }^{a}$ Maastricht University, \\ Maastricht, the Netherlands \\ E-mail: j.devries@cern.ch
}

Measurements of $b \rightarrow$ sll mediated electroweak penguin decays have recently shown tantalizing discrepancies with the Standard Model. However, improved and additional measurements are required to confirm or dismiss these observations. This talk discusses two recent results from the LHCb collaboration on such transitions.

The Eighth Annual Conference on Large Hadron Collider Physics-LHCP2020

25-30 May, 2020

online

${ }^{1}$ on behalf of the LHCb Collaboration.

* Speaker 


\section{Introduction}

In the search for physics beyond the Standard Model (SM), recent excitement has occured around a group of indirect measurements involving the transition of a b-quark to an s-quark and two leptons, called the 'flavour anomalies' (Fig. 1 (right)) [1]. These transitions are flavour-changing and neutral, and hence can only occur at loop-level Feynman diagrams in the SM, making them suppressed to about one part in $10^{-7}$ or smaller, and sensitive to new physics contributions in these loops. The heavy degrees of freedom associated with these loops can be integrated out and replaced by model-independent effective couplings (so-called Wilson coefficients). There are three key observables associated with $b \rightarrow$ sll transitions measured by the LHCb collaboration: branching fractions, branching fraction ratios (which are covered in a different talk) and angular observables. These measurements are performed as a function of $q^{2}=m_{\text {inv }}\left(\mu^{+} \mu^{-}\right)^{2}$, as different regions in $q^{2}$ are sensitive to different observables, and are affected by different theoretical uncertainties on the form factors (Fig. 1, left). This talk will focus on the two new measurements of $b \rightarrow$ sll transitions published by the LHCb Collaboration in early 2020 .
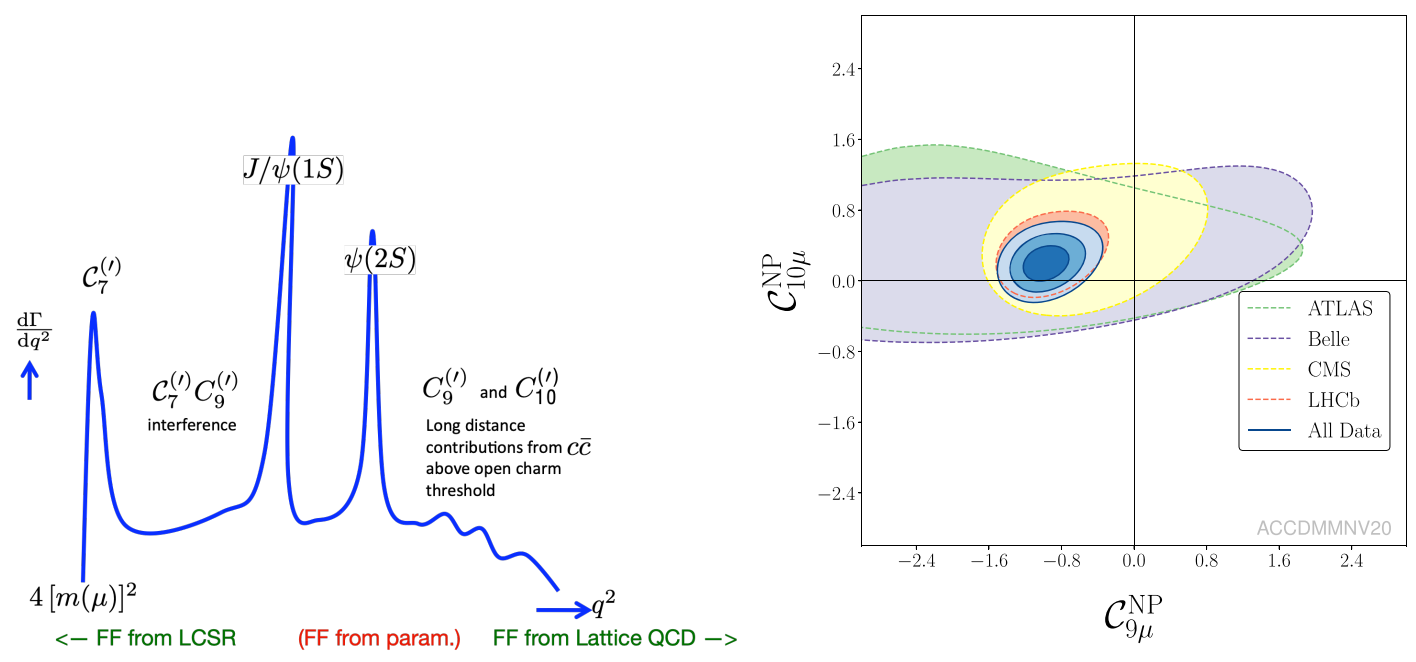

Figure 1: Left: behavior of the branching ratio as function of $q^{2}$. Indicated are the dominant Wilson coefficients and $c \bar{c}$ resonances. Right: one of many recent combinations of $O(100)$ measurements, indicating the tantalizing tension of the flavour anomalies with the Standard Model, which sits at $(0,0)[1]$.

\section{Angular analysis}

The measurement of $B_{d}^{0} \rightarrow K^{* 0} \mu^{+} \mu^{-}$is an angular analysis, meaning that besides the $q^{2}$, the measured branching ratio is parameterized as a function of three decay angles in the $B_{d}^{0}$ rest-frame: the angle between the two muons and the angle between the two decay products of the $K^{* 0} \rightarrow K^{+} \pi^{-}$ decay, and the angle between the di-muon and $K^{+} \pi^{-}$decay planes:

$$
\frac{d^{4} \Gamma\left[B_{d}^{0} \rightarrow K^{* 0} \mu^{+} \mu^{-}\right]}{d q^{2} d^{3} \vec{\Omega}}=\frac{9}{32 \pi} \sum_{i} I_{i}\left(q^{2}\right) f_{i}(\vec{\Omega}) .
$$


The angular coefficients $I_{i}\left(q^{2}\right)$ are functions of the $K^{* 0}$ polarisation amplitudes, which in turn depend on the Wilson coefficients and form factors for this decay. The branching ratio can be expressed in various bases, where the so-called $P_{i}$ basis is optimized to minimize the theory uncertainties. The previous LHCb measurement of $B_{d}^{0} \rightarrow K^{* 0} \mu^{+} \mu^{-}$found a local tension with the SM in the $P_{5}^{\prime}$ observable of 2.8 and 3.0 standard deviations in two adjecent bins of $4.0<q^{2}<6.0$ and $6.0<q^{2}<8.0 \mathrm{GeV}^{2} / \mathrm{c}^{4}$ respectively [2]. This 2020 update includes data taken by LHCb in 2016, effectively doubling the dataset [3].

A similar strategy compared to last analysis is employed, using the highly efficient LHCb muon trigger, and high vertex quality and impact parameter criteria. The latter is to ensure a reasonable decay length of the $B_{d}^{0}$ meson, separating it from random combinations of tracks from the primary interaction point. Particle identification techniques are used to reject backgrounds that peak in the invariant mass near the signal peak, most prominently from $\bar{\Lambda}_{b}^{0} \rightarrow \bar{p} K^{+} \mu^{+} \mu^{-}$and $B_{s}^{0} \rightarrow \phi(1020)\left[\rightarrow K^{-} K^{+}\right] \mu^{+} \mu^{-}$decays, where the proton (kaon) is misidentified as a pion. Multivariate techniques are employed to further suppress combinatorial backgrounds. Eight bins in $q^{2}$ are defined, avoiding the regions where $c \bar{c}$ resonances occur. These resonances, where no new physics is expected, are used to validate all analysis steps.

The angular distributions are affected by the efficiencies of selecting and reconstructing the data. These responses are obtained from high-statistics simulated events, and parameterized by 4D Legendre polynomials. Finally, a simultaneous maximum-likelihood fit is performed to the mass and the three decay angles, where the eight observables are shared among all the dataset years. An $\mathrm{S}$-wave component for the $\mathrm{K}^{+} \pi^{-}$system is taken into account in the invariant mass fit. The error on the fitted parameters is dominated by the statistical error, while the systematic errors are driven by the evaluation of the acceptance response, estimates of the peaking backgrounds mentioned above, and fit biases.

The new results for the $P_{5}^{\prime}$ observable are shown in Fig. 2 (left). The local tension in the same $q^{2}$ bins defined above have slightly shifted to 2.5 and 2.9 standard deviations, respectively. Even though the error on the datapoints has shrunk and form factor uncertainties have decreased, the sub-leading theory corrections have been stated more conservatively $[8,9]$.

Overall, the behavior of all eight observables has become more consistent. Varying the vector $\left(C_{9}\right)$ and axial vector $\left(C_{10}\right)$ Wilson coefficients, it is observed that the results are best explained by a single change of $C_{9}$ to be around -1 , with a significance of around three standard deviations. Similar results are obtained in various model-indepent combinations of $O(100)$ measurements (Fig. 1 (right)) [1]. This hints towards new vector contributions, such as leptoquarks, to explain these observations. 


\section{Decays to two leptons}

The decays of b-hadrons to two leptons also fall into the category of $b \rightarrow$ sll transitions and have even lower branching ratios in the SM due to helicity suppression,

$$
\operatorname{BR}\left(B_{q} \rightarrow l l\right) \propto\left|V_{\mathrm{tb}} V_{\mathrm{tq}}\right|^{2}\left[\left(1-\frac{4 m_{l}^{2}}{M_{B}^{2}}\right)\left|C_{S}-C_{S}^{\prime}\right|^{2}+\left|\left(C_{P}-C_{P}^{\prime}\right)+\frac{2 m_{l}}{M_{B}}\left(C_{10}-C_{10}^{\prime}\right)\right|^{2}\right] .
$$

These decays are sensitive to changes in $C_{10}$, and in addition to (pseudo)scalar contributions that lift the helicity suppression. As such their measurements provide unique constraints to the global coefficient landscape. The recent combination of $B_{q}^{0} \rightarrow \mu^{+} \mu^{-}$measurements [4], of which the LHCb measurement is the most precise [5], displays a branching ratio for the $B_{s}^{0}$ that is about two standard deviations below the SM. An update from LHCb including the full Run1+2 dataset is expected soon.

The decay of $B_{s}^{0}$ to two electrons is even more strongly helicity suppressed, such that the observation of a single event in the LHCb dataset would be a clear sign for new physics [6]. LHCb has recently published a search for this decay, where no events were observed (Fig. 2 (right) ) [7]. A limit on the branching ratio has been set at $\operatorname{BR}\left(B_{s}^{0} \rightarrow e^{+} e^{-}\right)<11.2 \times 10^{-9}$ at $95 \%$ confidence level, providing valuable constraints on (pseudo)scalar contributions.
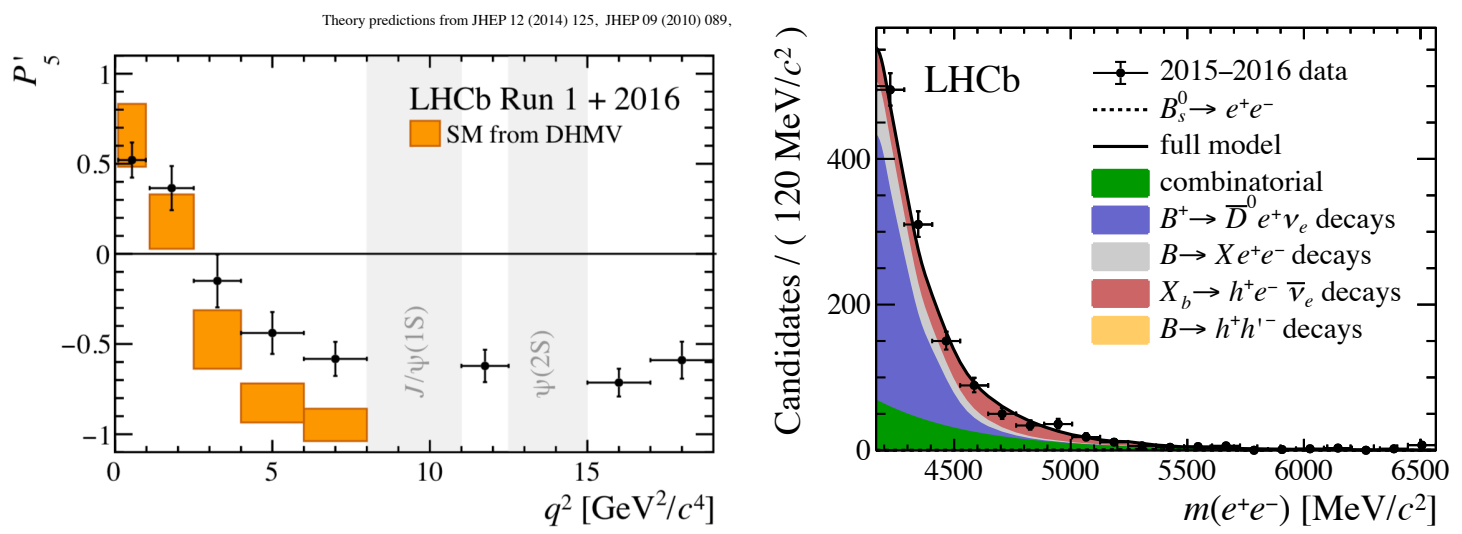

Figure 2: Left: the $P_{5}^{\prime}$ observable in bins of $q^{2}$ from Ref. [3], with the Standard Model expectation in orange. Right: result of the search for $B_{d, s}^{0} \rightarrow e^{+} e^{-}$[7].

\section{Conclusions}

Flavour physics is experiencing an exciting time. The flavour anomalies in $b \rightarrow s l l$ transitions display discrepancies with the SM, with various model-independent combinations reaching over four standard deviation significances (Fig. 1 (right)). Various decay channels provide essential complementary contributions to this picture. This presentation highlighted two recent results from the LHCb collaboration: the update of the angular analysis of $B_{d}^{0} \rightarrow K^{* 0} \mu^{+} \mu^{-}$and the search for $B_{s, d}^{0} \rightarrow e^{+} e^{-}$. Many more results are expected soon, and the community is looking forward to any conclusions the flavour anomalies will reach in the coming years. 


\section{References}

[1] M. Algueró, B. Capdevila, A. Crivellin, S. Descotes-Genon, P. Masjuan, J. Matias, M. NovoaBrunet, J. Virto, Eur. Phys. J. C80 (2020) 511 [arXiv:hep-ph/1903.09578]

[2] R. Aaij et al. (The LHCb Collaboration), J. High Energ. Phys. 02 (2016) 104 [arXiv:hep-ex/1512.04442]

[3] R. Aaij et al. (The LHCb Collaboration), Phys. Rev. Lett. 125 (2020) 011802 [arXiv:hep-ex/2003.04831]

[4] R. Aaij et al. (The LHCb Collaboration), LHCb-CONF-2020-002

[5] R. Aaij et al. (The LHCb Collaboration), Phys. Rev. Lett. 118 (2017) 191801 [arXiv:hep-ex/1703.05747]

[6] R. Fleischer, R. Jaarsma, G. Tetlalmatzi-Xolocotzi, J. High Energ. Phys. 05 (2017) 156 [arXiv:hep-ph/1703.10160]

[7] R. Aaij et al. (The LHCb Collaboration), Phys. Rev. Lett. 124 (2020) 211802 [arXiv:hep-ex/2003.03999]

[8] A. Bharucha, D. M. Straub, R. Zwicky, J. High Energ. Phys. 08 (2016) 98 (2016)

[9] W. Altmannshofer, D. M. Straub, Eur. Phys. J. C75 (2015) 382 